\title{
FLOTAÇÃO SELETIVA DE CROMITA COM CARBOXILMETILCELULOSE
}

\author{
C. A. M Baltar ${ }^{1}$; A.R.S.Medeiros ${ }^{2}$; M.M. Rodrigues $^{3}$ \\ ${ }^{1}$ Grupo de Tecnologia Mineral - Universidade Federal de Pernambuco; ${ }^{2}$ Mestranda, PPG Eng. Mineral, UFPE; \\ ${ }^{3}$ Graduanda em Engenharia de Minas da UFPE. \\ camb@ufpe.br - tiagoamf@yahoo.com.br - ailma.engminas@gmail.com
}

Artigo submetido em outubro/2012 e aceito em outubro/2012

\section{RESUMO}

O minério de cromo da Bahia é constituído por cromita $\left(\mathrm{FeO} . \mathrm{Cr}_{2} \mathrm{O}_{3}\right)$ e uma variedade de minerais silicatados de ganga, contendo íons $\mathrm{Ca}^{++}$e $\mathrm{Mg}^{++}$, entre outros. A flotação seletiva é bastante complicada devido às características superficiais similares dos componentes mineralógicos e só é possível com o uso de um reagente depressor. Nesse trabalho alguns dos principais depressores usados em flotação foram testados: silicato de sódio, amido e carboxilmetilcelulose (CMC). Os testes foram realizados em Tubo de Hallimond modificado.
Os resultados indicaram condições favoráveis à seletividade com o uso de CMC em concentrações acima de $100 \mathrm{mg} / \mathrm{L}$. A adsorção preferencial do CMC na superfície dos minerais de ganga foi atribuída à formação de sais carboxílicos, devido presença do grupo COO.Na na molécula do depressor e de íons $\mathrm{Ca}^{2+}$ e $\mathrm{Mg}^{2+}$ na superfície de alguns desses minerais.

PALAVRAS-CHAVE: flotação de cromita, depressor, carboxilmetilcelulose, amido, silicato de sódio.

\section{SELECTIVE FLOTATION OF CROMITE WITH CARBOXYLMETILCELULOSE}

\section{ABSTRACT}

The chrome ore from Bahia consists of chromite $\left(\mathrm{FeO} . \mathrm{Cr}_{2} \mathrm{O}_{3}\right)$ and gangue minerals such as magnetite, serpentine and pyroxene. The selective flotation is quite complicated due to similar mineralogical components' surface characteristics being only possible with the use of a depressant agent. In this work, it was tested some of the mains depressant used in flotation: sodium silicate, starch and Carboxymethyl cellulose (CMC). The flotation tests were conducted in a modified Hallimond Tube. The results indicated favorable conditions for selectivity with the use of $\mathrm{CMC}$ at concentrations above $100 \mathrm{mg} / \mathrm{L}$. The preferential adsorption of depressant on gangue minerals surface was attributed to the formation of carboxylic salts due the presence of group COO. $\mathrm{Na}$ at the $\mathrm{CMC}$ molecule and $\mathrm{Ca}^{2+}$ and $\mathrm{Mg}^{2+}$ ions on some of the gangue minerals surfaces.

KEY-WORDS: chromite flotation, depressing agents, Carboxymethyl cellulose, starch, sodium silicate. 


\section{FLOTAÇÃO SELETIVA DE CROMITA COM CARBOXILMETILCELULOSE}

\section{INTRODUÇÃO}

O minério de cromo da Bahia é constituído por cromita e por uma variedade de minerais silicatados de ganga, entre os quais a clorita, a hornblenda, a serpentina e o talco (Tabela 1):

Tabela 1 - Composição química de alguns dos minerais presentes no minério de cromo da Bahia.

\begin{tabular}{|l|c|}
\hline \multicolumn{1}{|c|}{ MINERAL } & COMPOSIÇÃO QUÍMICA \\
\hline cromita & ${\mathrm{FeO} . \mathrm{Cr}_{2} \mathrm{O}_{3}}^{\mathrm{Fe}_{3} \mathrm{O}_{4}}$ \\
\hline magnetita & $(\mathrm{Mg}, \mathrm{Fe})_{3}(\mathrm{Si}, \mathrm{Al})_{4} \mathrm{O}_{10}(\mathrm{OH})_{2} \cdot(\mathrm{Mg}, \mathrm{Fe})_{3}(\mathrm{OH})_{6}$ \\
\hline clorita & $(\mathrm{Ca}, \mathrm{Na})_{2-3}(\mathrm{Mg}, \mathrm{Fe}, \mathrm{Al})_{5}\left(\mathrm{Al}, \mathrm{Si}_{8} \mathrm{O}_{22}(\mathrm{OH}, \mathrm{F})_{2}\right.$ \\
\hline hornblenda & $(\mathrm{Mg}, \mathrm{Fe}, \mathrm{Ni}, \mathrm{Al}, \mathrm{Zn}, \mathrm{Mn})_{2-3} \mathrm{Si}_{2} \mathrm{O}_{5}(\mathrm{OH})_{4}$ \\
\hline serpentina & $\mathrm{Mgg}_{3} \mathrm{Si}_{4} \mathrm{O}_{10}(\mathrm{OH})_{2}$ \\
\hline talco & \\
\hline carbonatos & \\
\hline
\end{tabular}

A flotação seletiva é bastante complicada devido às características superficiais similares dos componentes mineralógicos. Observa-se na Tabela 1 que o ferro está presente na superfície da cromita e, também, na superfície dos minerais de ganga magnetita, clorita, hornblenda e serpentina.

Além disso, a ação da água sobre a superfície dos minerais provoca a liberação de espécies iônicas como $\mathrm{Mg}^{+2}, \mathrm{Ca}^{+2}, \mathrm{Fe}^{+3}$ e $\mathrm{Al}^{+3}$, entre outras. Esses íons em solução, em geral, participam ativamente do processo de flotação, podendo: (1) alterar a carga elétrica superficial (RAO et al., 1988); (2) provocar a precipitação do coletor; (3) competir com o íon coletor pelos sítios disponíveis na superfície (BALTAR e CUNHA, 2002); e (4) contribuir para a estabilização da espuma (QUINN et al., 2007).

A influência de espécies catiônicas na flotação de diferentes minerais com amina foi estudada por RAO et al. (1988), BALTAR e CUNHA (2002), e PINHEIRO et al. (2010). RAO et al. (1988) conseguiram melhorar os resultados de uma flotação de pirocloro após a remoção de íons $\mathrm{Ca}^{2+}$ da água de reciclagem. BALTAR e CUNHA (2002) observaram que a presença de apenas $5 \mathrm{ppm}$ de $\mathrm{Al}^{3+}$ reduziu a recuperação de feldspato de $95 \%$ para $50 \%$, enquanto o efeito depressor do $\mathrm{Ca}^{2+}$ e do $\mathrm{K}^{+}$só foi percebido para concentrações maiores do que 2000 ppm. PINHEIRO et al. (2010) elevaram a recuperação do quartzo de $14 \%$ e $84 \%$ após a redução dos

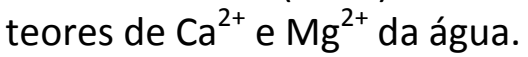

Diante dessas dificuldades, torna-se evidente a necessidade do uso de um reagente depressor. Essa categoria de reagente atua no sentido de inibir a adsorção do coletor em 
determinadas superfícies minerais (BALTAR, 2010). Dentre os mais comuns estão o silicato de sódio, o amido de milho e o carboxilmetilcelulose (CMC). O primeiro é um depressor inorgânico bastante utilizado na depressão de silicatos. O processo de hidrólise do silicato de sódio é complexo, gerando uma variedade de espécies iônicas (monoméricas e poliméricas) e coloidais, entre as quais: $\mathrm{HSiO}_{3}{ }^{-}, \mathrm{SiO}_{3}{ }^{2-},\left(\mathrm{mSiO}_{3} \cdot n \mathrm{niO}_{2}\right)^{2 \mathrm{~m}-}, \mathrm{H}_{2} \mathrm{SiO}_{3}$ (aq.), além de colóides nas formas de $\mathrm{SiO}_{2}$ e $\mathrm{Na}_{2} \mathrm{O}_{\gamma} \mathrm{SiO}_{2}$ (SILVA, 2011; MARINAKIS et al., 1985; QI et al., 1993). A adsorção de qualquer uma dessas espécies na superfície impede a adsorção do coletor. $O$ amido de milho é um conhecido depressor de hematite na flotação reversa de minério de ferro (ARAÚJO et al., 2005) e adsorve-se por meio de sitos hidróxido metálicos da superfície (LIU et al., 2000; LASKOWSKI et al., 2007; BALTAR, 2010). Por sua vez, o CMC é obtido a partir de uma reação entre a celulose e o ácido monocloroacético (ou seus sais de sódio) resultando na presença de grupos metil carboxila de sódio na molécula do CMC (BALTAR, 2010).

O processo de beneficiamento de minério de cromo no Brasil é baseado em métodos gravimétricos, predominando o uso de espirais. Esses processos geram uma grande quantidade de finos com teor médio de $10 \% \mathrm{Cr}_{2} \mathrm{O}_{3}$.

O estudo teve por objetivo procurar condições favoráveis à seletividade na flotação catiônica da cromita, testando depressores usuais para minerais de ganga silicatada e carbonatada.

\section{EXPERIMENTAL}

\section{$\underline{\text { Material }}$} FERBASA.

a) Amostra - As amostras de cromita e dos minerais de ganga foram fornecidas pela

b) Principais Equipamentos - Os testes de flotação foram realizados em Tubo de Hallimond modificado com diâmetro de $3,5 \mathrm{~cm}$ e constituído de três partes (Figura 1); agitador magnético QUIMIS modelo 241-2; e um pHmetro digital da DIGIMED, modelo DM-22.

c) Reagentes - Usou-se uma dodecilamina produzida pela SIGMA-ALDRICH, um amido de milho comercial, um CMC (SIGMA-ALDRICH), um silicato de sódio (VETEC) e o hidróxido de sódio (ambos da QUIMEX).

\section{Metodologia}

Testes de Flotação

Os ensaios de microflotação foram realizados em tubo de Hallimond modificado (Figura 1) alimentado com uma vazão de nitrogênio de $80 \mathrm{~mL} / \mathrm{min}$. Usou-se 1 grama de cromita pura (faixa granulométrica $100 \%$ menor que $74 \mu \mathrm{m}$ ) ou, conforme o caso, da amostra representativa do rejeito da FERBASA. O pH foi fixado em 8,0 usando-se o hidróxido de sódio $(\mathrm{NaOH})$ como regulador. Como coletor usou-se a dodecilamina, mantendo-se a concentração de $10^{-3} \mathrm{M}$. O tempo de condicionamento foi de 1 minuto, e o tempo de flotação foi de 2 minutos. Após, cada teste de flotação, os produtos (flotado e não-flotado) foram filtrados, secados e pesados. 


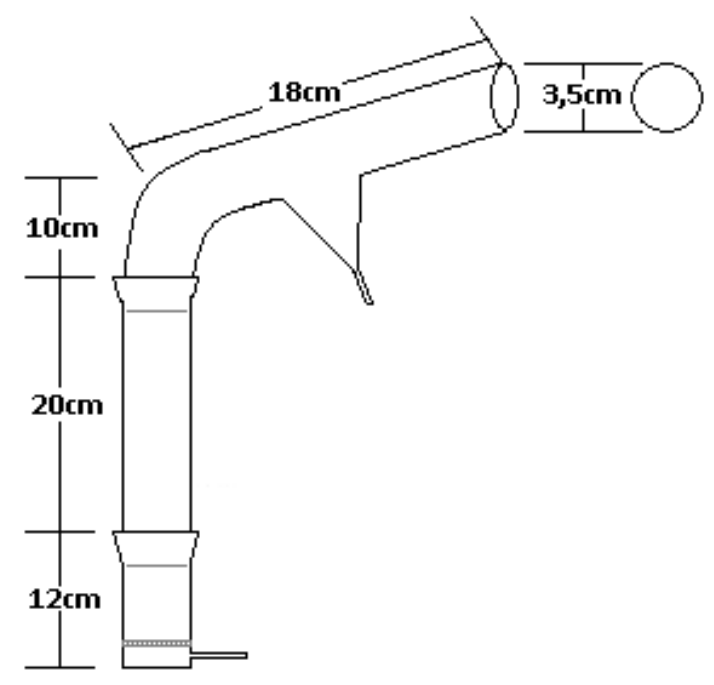

Figura 1 - Ilustração com as dimensões do Tubo de Hallimond modificado usado nos testes de flotação.

\section{RESULTADOS E DISCUSSÃO}

Os resultados obtidos nos testes de flotação são apresentados nas Figuras 2 a 4, onde se pode comparar a influência dos diferentes depressores na flotação catiônica da cromita e da ganga.

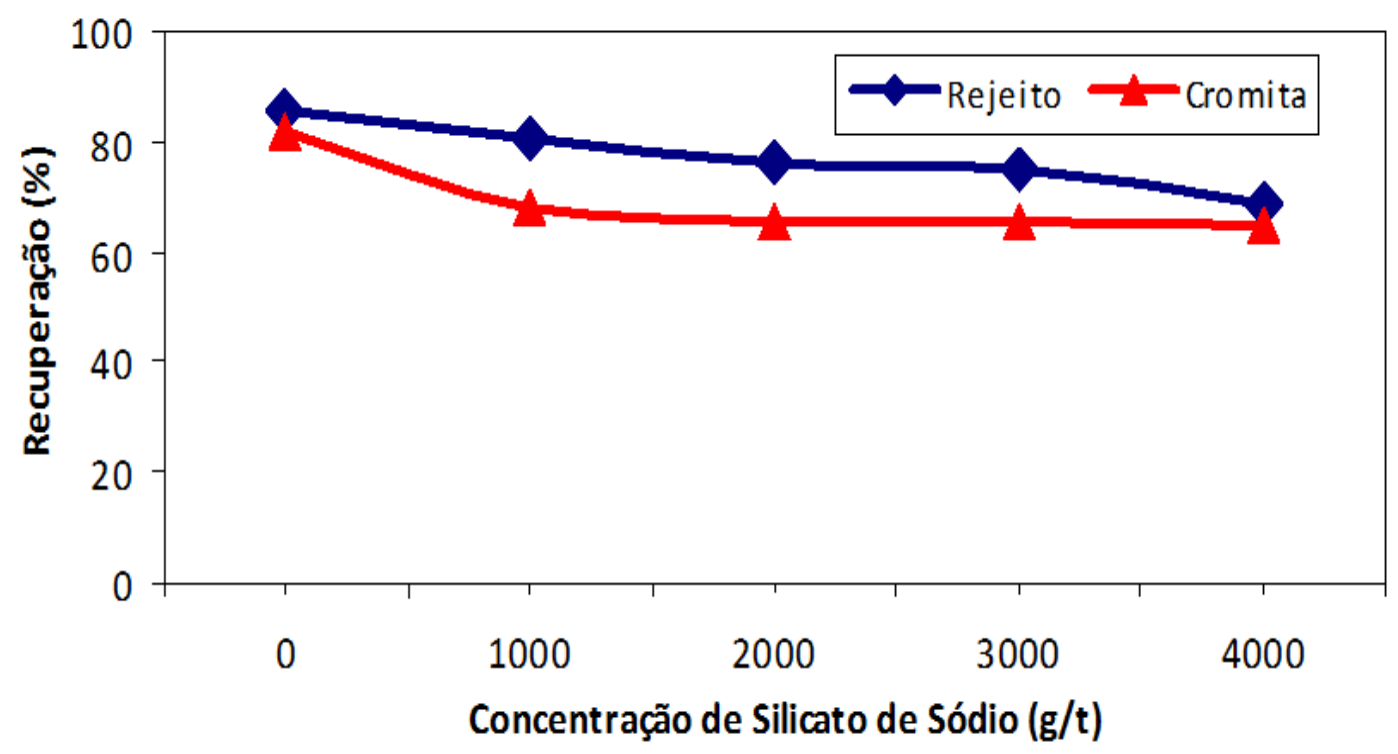

Figura 2 - Influência da concentração do silicato de sódio (SS) na seletividade da cromita em relação aos minerais de ganga.

Os resultados mostrados na Figura 2 indicam uma influência discreta e pouco seletiva do silicato de sódio com atuação maior na superfície da cromita. 


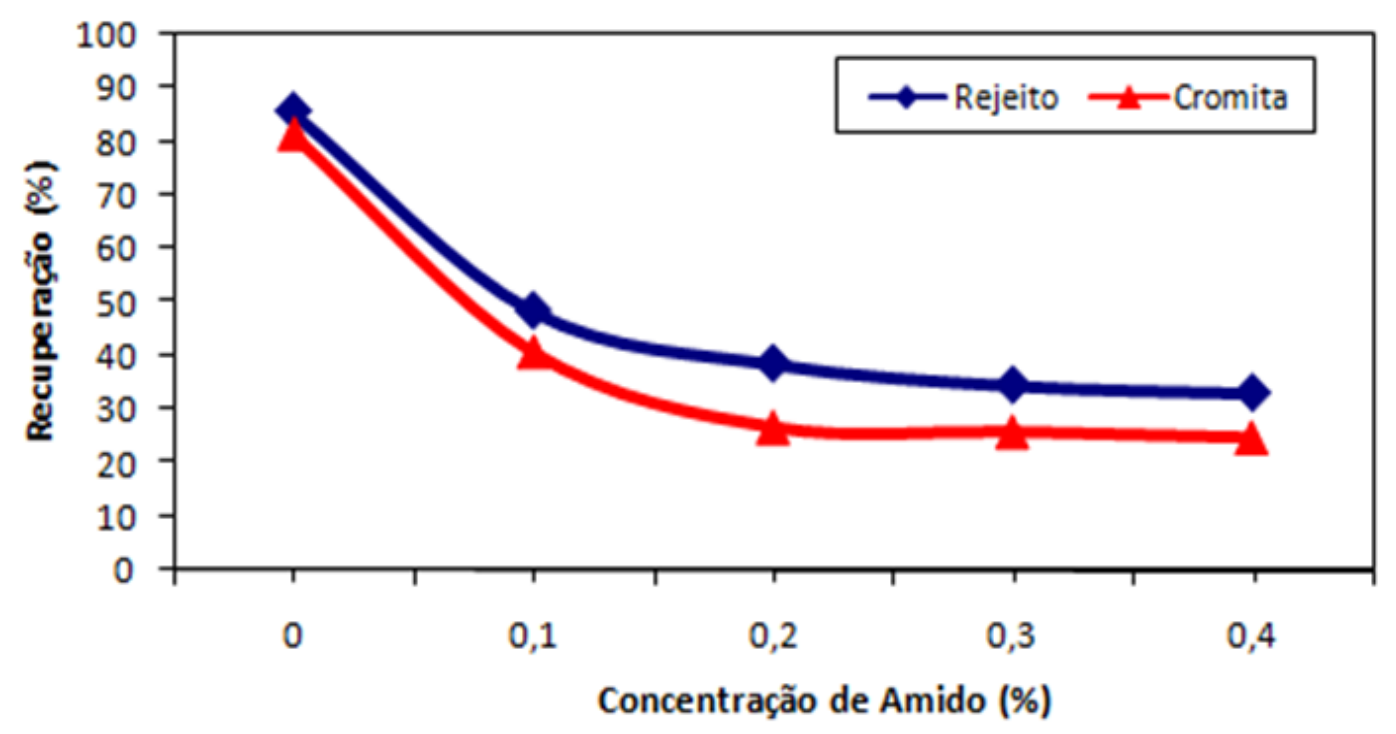

Figura 3 - Influência da concentração de amido na seletividade da cromita em relação aos minerais de ganga.

Os resultados da Figura 3 mostram uma considerável influência do amido na depressão de ambas as amostras. Com apenas $0,2 \%$ de amido, a recuperação da ganga cai de $85,8 \%$ para $38,6 \%$, enquanto a da cromita é reduzida de $81,5 \%$ para $26,8 \%$. Portanto, a ação do amido é não seletiva, deprimindo indistintamente a cromita e os minerais de ganga. Esse fato sugere a participação do íon $\mathrm{Fe}^{3+}$, presente na superfície da cromita, magnetita, clorita, hornblenda, serpentina e minerais óxidos de ferro, como provável sítio para a adsorção do amido.

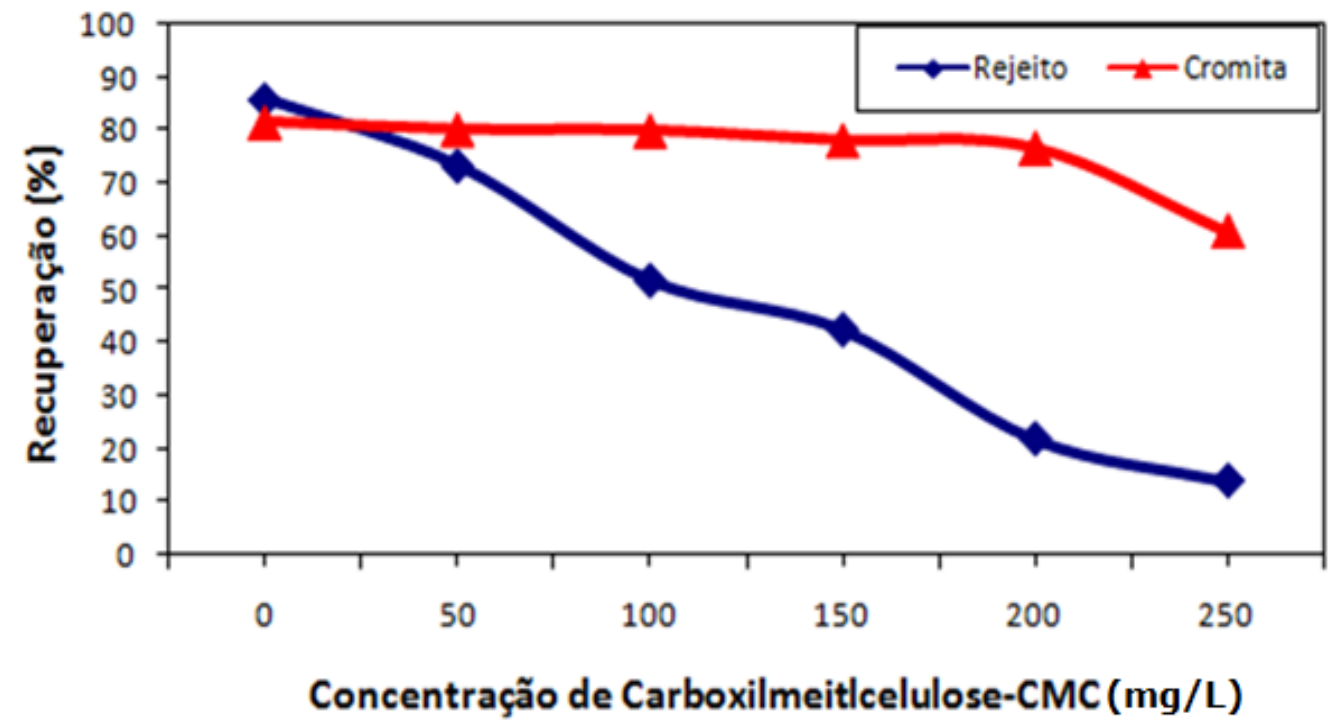

Figura 4 - Influência da concentração do CMC na seletividade da cromita em relação aos minerais de ganga.

Os resultados da Figura 4 sugerem a possibilidade de seletividade na flotação da cromita com o uso do CMC a partir de concentrações de $100 \mathrm{mg} / \mathrm{L}$. A seletividade se deve à 
presença do grupo carboxílico (COO) na molécula do CMC (Figura 5). Esse grupo iônico tem grande afinidade pelos alcalinos terrosos presentes na maioria dos minerais de ganga com os quais forma sais praticamente insolúveis.

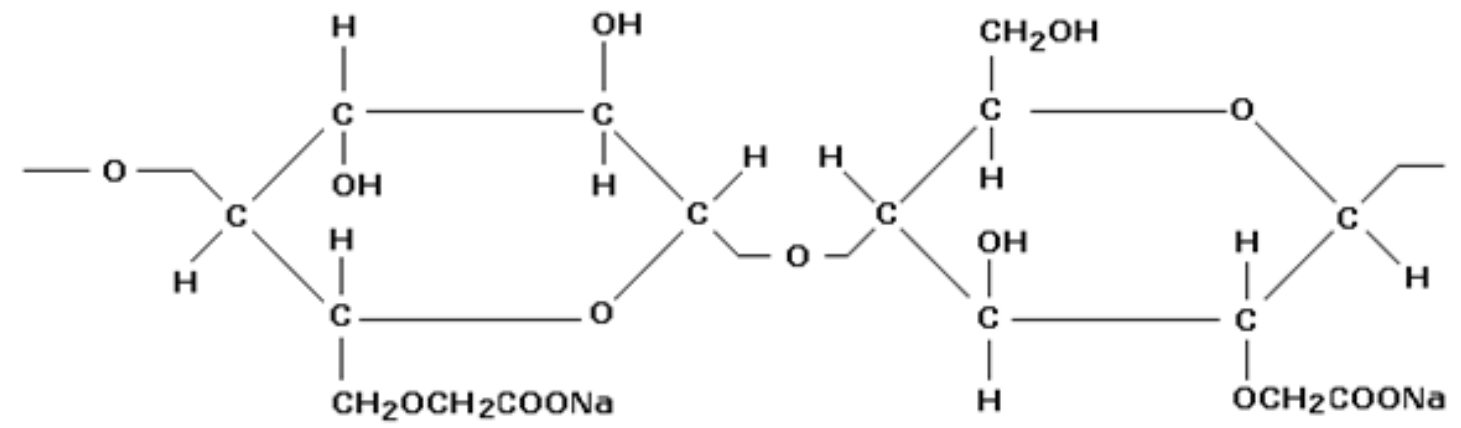

Figura 5 - Molécula do carboxilmetilcelulose com o grupo carboxílico resultante da reação de eterificação da celulose.

\section{CONCLUSÕES}

Procurou-se identificar as condições mais favoráveis à seletividade, na flotação catiônica da cromita, testando-se depressores tradicionalmente utilizados para minerais de ganga à base de silicatos e de carbonatos.

O silicato de sódio teve uma atuação discreta, e não seletiva, na faixa de concentração e $\mathrm{pH}$ testada. Por sua vez, o amido de milho deprimiu indistintamente a cromita e os minerais de ganga. Isso se deve, provavelmente, à presença comum de ferro na superfície da cromita e dos minerais de ganga.

O CMC apresentou uma ação depressora seletiva para os minerais de ganga. $O$ mecanismo de adsorção sugerido foi a formação de um sal praticamente insolúvel na superfície dos minerais com $\mathrm{Mg}^{2+}$ e $\mathrm{Ca}^{2+}$ por meio do grupo carboxílico da molécula do depressor.

\section{AGRADECIMENTO}

Os autores agradecem ao CTMineral/CNPq pelo apoio financeiro ao projeto "Aproveitamento de Finos de Cromita por Flotação" e pela bolsa de Iniciação Científica. À CAPES pela bolsa de mestrado de uma das autoras.

\section{REFERÊNCIAS BIBLIOGRÁFICAS}

ARAÚJO, A.C.; VIANA, P.R.M.; PERES, A.E.C. Reagents in iron ores flotation. Minerals Engineering, 18, 219-224, 2005. 
BALTAR, C.A.M.; CUNHA, A.S.F. Influência de espécies catiônicas na flotação de feldspato com amina. In.: Encontro Nacional de Tratamento de Minérios e Metalurgia Extrativa, Anais, v1. BALTAR, C.A.M.; BARBOSA, J.P. e OLIVEIRA, J,C.S. (editores). Recife, p. 234-240, 2002.

BALTAR, C.A.M. Flotação no Tratamento de Minérios. 2a edição, Editora Universitária da UFPE, 238 p., 2010.

LASKOWISKI, J.S.; LIU, Q.; O'CONNOR, C.T. Current understanding of the mechanism of polysaccharide adsorption at the mineral/aqueous solution interface. International Journal of Mineral Processing, 84, 59-68, 2007.

LIU, Q.; ZHANG, Y.; LASKOWSKI, J.S. The adsorption of polysaccharides onto mineral surface: an acid/base interaction. International Journal of Mineral Processing, 60 (3-4), 229-245, 2000.

MARINAKIS, K. I.; SHERGOLD, H. L. Influence of sodium silicate addition on the adsorption of oleic acid by fluorite, calcite and barite. International Journal of Mineral Processing, 14, p.177193, 1985.

PINHEIRO, V.S.; BALTAR, C.A.M.; LEITE, J.Y.P. Influência da qualidade da água na flotação de quartzo com amina. HOLOS, 3, p. 28-36, 2010.

Qi, G.W.; Klauber, C.; Warren, L.J. Mechanism of action of sodium silicate in the flotation of apatite from hematite. International Journal of Mineral Processing, 39, 251-273, 1993.

QUINN, J.J.; KRACHT, W.; GOMEZ, C.O.; GAGNON, C.; FINCH, J.A. Comparing the effect of salts and frother (MIBC) on gas dispersion and froth properties. Minerals Engineering, 20, p. 12961302, 2007.

RAO, S.R.; ESPINOSA-GOMEZ, R.; FINCH, J.A.; BISS, R. Effects of water chemistry on the flotation of pyrochlore and silicate minerals. Minerals Engineering, 1 (3), 189-202.

SILVA, J.P.P. Mecanismo de ação do silicato de sódio como depressor em flotação. Dissertação de Mestrado, Programa de Pós-Graduação em Engenharia Mineral, Universidade Federal de Pernambuco. 67 p., 2011. 